\title{
ENTORNOS VIRTUALES DE APRENDIZAJE COMO ESPACIO COMPLEMENTARIO EN LA FORMACIÓN INICIAL DE PROFESORES
}

\author{
Implementation of Virtual Learning Environments as \\ a Complementary Space in Initial Teacher Training
}

\section{Cristian Clavijo-Gallo ${ }^{1}$}

cristian.clavijo@udea.edu.co

Daniel Andrés Quiróz-Vallejo1

daniel.quirozv@udea.edu.co

Edgar Johan Arboleda-Mira'

edgar.arboleda@udea.edu.co

Jaime Andrés Carmona-Mesa ${ }^{1}$

jandres.carmona@udea.edu.co

${ }^{1}$ Universidad de Antioquia. Colombia

Recibido: $12 / 03 / 2021$

Revisado: 30/04/2021

Aprobado: $7 / 05 / 2021$

Preprint: 7/06/2021

Publicado: 15/07/2021

\section{Resumen}

El objetivo de este artículo es describir las proyecciones de uso de los entornos virtuales de aprendizaje (EVA) como espacios complementarios de formación dentro de los cursos orientados a la integración de las TIC en procesos educativos de la Facultad de Educación de la Universidad de Antioquia (Medellín, Colombia). Para ello se adopta un enfoque cualitativo fundamentado en una revisión documental de los programas de curso y en entrevistas semiestructuradas con los profesores que orientan dichos espacios. Los resultados se reportan a partir de cuatro categorías: roles, interactividad, habilidades y aptitudes, y potencial educativo de los EVA. Dentro de los hallazgos se destaca que los roles de los actores educativos cambian al interactuar en un EVA; la interacción que posibilitan estos entornos da paso a nuevas formas de relacionarse. Se hace hincapié en el componente de autonomía que se requiere de los futuros profesores para participar en estos espacios, además de las necesidades de formación en tecnología para que los procesos educativos en un EVA se desarrollen de manera exitosa.

Palabras clave: formación inicial de profesores, entornos virtuales de aprendizaje, roles, competencias, interactividad, autonomía.

\section{Abstract}

The objective of this article is to describe the projections of uses of Virtual Learning Environments (VLEs) as Complementary Training Spaces (CTS) within the courses oriented to the integration of ICTs in educational processes of the School of Education at the University of Antoquia. (Medellin, Colombia). For this purpose, a qualitative approach is adopted. A documentary review of course syllabi was carried out and semi-structured interviews were conducted with the teachers who guide the courses. The results are reported from four categories: roles, interactivity, competencies, and educational potential of VLEs. The findings highlight that the roles of the educational actors change when interacting in a VLE; the interaction made possible by these environments gives place to new ways of relating to others. Emphasis is placed on the autonomy component required of future teachers to participate in these spaces, in addition to the need for training in technologies so that the educational processes in an VLE can be developed successfully.

Keywords: autonomy, competencies, interactivity, pre-service teacher, roles, virtual learning environments. 


\section{Introducción}

En los últimos 15 años, el uso masificado de las tecnologías de la información y comunicación (TIC) en educación superior se ha extendido por las diferentes universidades de Latinoamérica. En el caso de los programas de formación inicial de profesores (FIP), las instituciones han adoptado diversas iniciativas para materializar su uso, entre ellas se identifican los entornos virtuales de aprendizaje (EVA) usados tanto enA la educación virtual como de forma complementaria a la educación presencial (Hrastinski, 2019).

Esta investigación se centrará en el papel de los EVA como espacios complementarios de formación (ECF) a los programas presenciales. Los EVA son plataformas de educación virtual en las que los contenidos del curso se manejan a través de una interfaz consistente y estándar (Phungsuk et al., 2017). El uso de los EVA como ECF en FIP ha sido objeto de estudio de diversas investigaciones que integran redes sociales (Quiroz-Vallejo et al., 2020) y sistemas de gestión del aprendizaje (Durak, 2019), las cuales presentan conclusiones favorables para considerar los EVA como alternativas con un amplio potencial educativo (Arroyo-Vera et al., 2018).

En la región se reconoce la relevancia de fortalecer las habilidades digitales en la FIP y aportar al desarrollo de estrategias para un uso consciente de EVA. Sin embargo, Fainholc (2016) halla debilidades en el proceso en cuanto a la cobertura de Internet y el uso eficiente de las TIC. Por otra parte, investigadores como Hue Dung (2020) sugieren el uso de EVA como oportunidades para fortalecer el ejercicio profesoral y profesional, lo que puede aportar a la resignificación del rol que desempeñan profesores, estudiantes y los recursos tecnológicos en los procesos de enseńanza y aprendizaje.

Si bien los EVA se identifican como recursos con amplio potencial educativo, su uso por parte de profesores requiere un mayor interés investigativo en aspectos como el uso en la formación profesional (Badilla et al., 2017), así como en la formulación de consideraciones teóricas y educativas que atiendan a las limitaciones técnicas en cuanto al acceso y disponibilidad de la red (Granados et al., 2017). Además, se identifica que el diseño de EVA como ECF presenta dificultades a la hora de distribuir y organizar las actividades para el contexto virtual y el presencial (Hrastinski, 2019). Lo anterior refleja la necesidad de ampliar las experiencias formativas que posibiliten su uso como ECF y aportar a la solución de las dificultades previamente indicadas.

En este sentido, la literatura revela que el interés en torno a los EVA está relacionado con satisfacer diversas necesidades como la identificación de las posibles interacciones que posibilitan los EVA entre profesores y profesores en formación, la integración de metodologías con carácter híbrido en la formación de profesores (González et al., 2013), y la identificación de las características cognitivas y sociales a considerar en la virtualidad para favorecer el trabajo colaborativo, la creación de redes académicas, la autonomía y la construcción conjunta de conocimiento. De la misma manera, investigadores como Ayil-Carrillo (2018) sugieren que la aplicación de ejercicios de repasos académicos en los ECF incrementa el rendimiento académico en pruebas, flexibiliza el trabajo y permite a los estudiantes avanzar a su ritmo y estilo de aprendizaje.

Lai y Xue (2012) reportan que el uso de EVA como ECF permite el desarrollo de acciones de formación que no finalizan en el lugar físico del aula de clase, dado que se amplía hacia espacios de interacción virtual en los cuales el flujo de discusiones es constante. En este sentido, identificar las formas de uso de EVA en instituciones de educación superior es una problemática relevante que debe ser investigada, en tanto posibilita proyectar rutas de acción para potenciar las habilidades digitales de los estudiantes. Por lo anterior, el objetivo de este artículo es describir las proyecciones de uso de EVA como espacios complementarios en cursos de TIC de los programas de formación inicial de profesores de la Facultad de Educación de la Universidad de Antioquia a través del análisis de los programas de curso y entrevistas a profesores que los dirigen. 


\section{Marco conceptual}

Los EVA se entienden como aquellos espacios educativos que agrupan un conjunto de herramientas TIC orientadas a la integración didáctica y la gestión de conocimiento (Ayil-Carrillo, 2018). Resultan útiles por su flexibilidad en cuanto a los roles de quienes interactúan y por favorecer la motivación de los participantes (Hrastinski, 2019). De igual forma, beneficia la interactividad asincrónica, en contraste con los espacios presenciales que en ocasiones resultan limitados y condicionados por diversos factores como la infraestructura disponible (Granados et al., 2017).

En este sentido, se estructuran dos modalidades que modifican los usos y los propósitos de los EVA. La primera modalidad sugiere un entorno completamente virtual (e-learning) y su propósito principal es ofrecer una experiencia educativa completamente virtual (Hue Dung, 2020). La segunda modalidad se conoce como B-learning y ofrece multiplicidad de experiencias tanto virtuales como presenciales estableciendo interacciones entre estudiantes y profesores (Llorente-Cejudo \& Cabero, 2008). Por otro lado, se enfatiza la relevancia que presenta la virtualidad como ECF en experiencias presenciales, dado que permite ampliar discusiones y gestionar información de contenidos en el trabajo colaborativo que se presenta en un curso (Borba et al., 2018).

De igual manera, en el B-learning se incentivan retos para cada uno de los actores implicados en el desarrollo de un curso: por un lado, los profesores deben plantearse nuevas formas de orientar el proceso debido a la flexibilización de las actividades y compromisos que se plantean, así como a las formas de evaluar y solucionar las dificultades que emergen en el proceso de aprendizaje de los estudiantes (Monsalve \& Amaya, 2014). A su vez, al estudiante se le exige una capacidad de regular el flujo de información y el contenido, y se incentivan habilidades metacognitivas que le permitan planear la ruta más eficaz para aprender, y así ampliar sus capacidades mentales y disminuir la carga cognitiva que proviene del alto flujo de información (Badilla et al., 2017).
En la literatura se reportan coincidencias referentes a los puntos de análisis de los estudios sobre EVA en la formación de profesores: (1) las habilidades y aptitudes que los futuros profesores deben tener para el uso del EVA, (2) los roles que se posibilitan entre profesores y futuros profesores, y (3) la interactividad de la plataforma con relación a la ubicuidad que ofrece. En adelante se profundizará en cada una de estas categorías.

\subsection{Habilidades y aptitudes}

En la actualidad, tanto profesores en ejercicio como futuros profesores tienen la necesidad de aprender distintas habilidades para desarrollar su ejercicio profesional. En particular, las habilidades relacionadas con el uso de recursos educativos digitales son importantes porque posibilitan participar de EVA con amplias posibilidades y características para implicar a los estudiantes en actividades que favorecen la construcción de conocimiento (Manrique-Losada et al., 2020). En este sentido, las habilidades y aptitudes que favorece la integración de los EVA como ECF en cursos de formación de profesores serán entendidas como los distintos aprendizajes que los futuros profesores adquieren gracias a interactuar en EVA.

De acuerdo con lo anterior, autores como García-Cabrero et al. (2018) proponen agrupar las habilidades que adquieren los profesores que participan en EVA en tres dimensiones: la previsión del proceso de enseñanza y aprendizaje, que incluye competencias como plantear el enfoque de los cursos, la planeación, el diseño de experiencias de aprendizaje, y el dominio en el uso y selección de TIC con finalidad educativa. Por otra parte, la dimensión conducción del proceso de enseñanza y aprendizaje incluye capacidades como gestionar los procesos de enseñanza, llevar a cabo la interacción didáctica, o usar canales de comunicación adecuados según los contextos. Finalmente, la dimensión valoración del impacto del proceso de enseñanza y aprendizaje se refiere a la capacidad de los docentes para usar formas adecuadas a la hora de valorar los procesos educativos y para implementar estrategias que favorezcan el aprendizaje autorregulado y colaborativo. 
En coherencia con lo anterior, autores como Cassidy (2016) declaran la relevancia de desarrollar en la FIP habilidades y aptitudes como la metacognición, valorar la calidad de los recursos educativos digitales para una mejor selección y uso, y tener en cuenta asuntos como la usabilidad y accesibilidad de los EVA. Por otra parte, los EVA combinan diferentes recursos educativos de distintas naturalezas, por lo cual también contribuyen a adquirir habilidades y aptitudes para gestionarlos (Zangara, 2018).

Finalmente, Arias et al. (2014) referencian una serie de habilidades digitales que se favorecen en los EVA, como el saber-hacer recursos educativos o softwares específicos de las disciplinas y la capacidad de gestionarlos, desarrollarlos y modificarlos. Sin embargo, su mayor énfasis se hace en la necesidad de identificar cómo y cuándo usar estos recursos en los EVA. En síntesis, las habilidades y aptitudes que se favorecen en los EVA son un aspecto importante a analizar en la literatura debido a la creciente demanda del uso de habilidades digitales en el campo educativo.

\subsection{Roles}

La educación presencial se ha caracterizado tradicionalmente por estar centrada en la figura del profesor, quien es considerado una autoridad y ocupa un rol dominante en el proceso educativo (Echeverry \& Jaramillo, 2017). Por el contrario, la educación virtual tiende a favorecer la apertura de los contenidos y ofrece facilidades de uso de las plataformas con interacción sincrónica y asincrónica (Lai \& Xue, 2012). En este contexto se evidencia que la educación virtual y presencial tienen particularidades relevantes, las cuales se mezclan en el caso de la integración de EVA como ECF. Ante este amplio panorama de posibilidades, los profesores se ven en la necesidad de adecuar sus roles en función de las diversas opciones que se presentan en los escenarios educativos (Hue Dung, 2020).

Respecto a los retos referentes a los roles de los profesores, Álvarez et al. (2009) proponen tres direcciones para este ajuste: el rol de planear y diseńar acciones educativas, el rol social y, por último, el rol educativo. En cambio, Borba et al. (2018) apuntan al estudio de los roles en EVA a partir de dos categorías: cómo los profesores, tutores y estudiantes producen materiales didácticos interactivos y cómo las tecnologías digitales en sí mismas juegan un papel en la enseñanza de cursos a distancia.

En suma, las diferentes propuestas para el análisis de los roles intentan generar una adecuación de las funciones de profesores y estudiantes en los EVA. Los profesores tienden a convertirse en guías del aprendizaje más que en transmisores de información, dado que esta ya suele encontrarse en el EVA. En cambio, los estudiantes toman una posición activa en su proceso educativo, siendo los gestores de sus espacios y tiempos para participar de las actividades del curso. De este modo, los roles se comprenden como las posibilidades de interacción entre profesores y estudiantes que son ofrecidas por el EVA (Quiroz-Vallejo et al., 2020) y como las posibilidades de interacción entre los actores educativos y los recursos tecnológicos utilizados.

\subsection{Interactividad}

La interactividad se puede comprender como el intercambio entre usuarios y sistemas virtuales. También se considera que la interactividad es la medida en que los usuarios pueden participar en la modificación de forma y contenido de un entorno en tiempo real (Zangara, 2018). Por tanto, la interactividad se refiere a la relación entre dos o más individuos que es mediada por un sistema que dinamiza la comunicación, brinda oportunidades de aprendizaje y permite adquirir información, así como acceder y modificar contenido hipermedia e hipertextual (Vixtha-Vázquez, 2017).

En el caso particular de los EVA, se reportan como significativas para su análisis la interactividad contextual no inmersiva y la interactividad contextual inmersiva (Veraszto \& García, 2011). La interactividad contextual no inmersiva permite al usuario interactuar en un EVA que presenta un entorno completo de trabajo, con los elementos necesarios para el desarrollo de actividades de aprendizaje, lo cual facilita una funcionalidad proactiva frente a los contenidos 
seleccionados por los profesores (Díaz-Gutiérrez \& Valderrama-García, 2018). Por su parte, la interactividad contextual inmersiva, aparte de facilitar la navegación proactiva mediante el entorno completo de trabajo, propicia que los estudiantes formen parte de la construcción del EVA, lo que les posibilita autogestionar el contenido, transformar la distribución y presentación de la información y construir su ruta de aprendizaje propia (Rodríguez-Ortiz \& Sosa-Neira, 2018).

Por otra parte, la interactividad también posee tipología. Autores como Sutcliffe y Hart (2017) ven dos tipos de interactividad: una orientada al objeto, que se refiere a la disposición de acciones activadas por periféricos (p. ej. atajos de teclado y comandos) y otra denominada interactividad de apoyo, que tiene que ver con la configuración de los menús, que son acompañados por sistemas de ayuda (p. ej. lector de pantalla). Estas características se enfocan desde un ámbito del diseńo del EVA que favorece o limita la navegabilidad. Vixtha-Vázquez (2017) reseña que existen otras características según el tipo de contenido (interactividad de actualización), las cuales se refieren al diálogo entre el usuario y el contenido en cuanto a la evaluación constante y la exigencia de actualización, la solución de fallos y la renovación de contenidos.

\section{Método}

En este estudio se desarrolló una revisión documental de los programas de curso ofrecidos entre 2018 y 2020 y entrevistas semiestructuradas a los profesores responsables de estos espacios, relacionados con la integración de las TIC en educación. El estudio se desarrolló en los programas destinados a formar profesores de la Facultad de Educación de la Universidad de Antioquia (Medellín, Colombia). Con el objetivo de describir las proyecciones de los usos de EVA como espacios complementarios en cursos de TIC de los programas de formación inicial de profesores en la Facultad de Educación de la Universidad de Antioquia, se realizó una triangulación intramétodos de la revisión documental de los programas de curso y de la transcripción de las entrevistas semiestructuradas (Flick, 2014). El análisis de la información se desarrolló por medio de la técnica de análisis de contenido exploratorio y descriptivo (Arbeláez \& Onrubia, 2014).

\subsection{Recolección de la información}

En la fase de recolección de la información se utilizaron dos técnicas: análisis documental y entrevistas semiestructuradas para analizar de manera tanto oral como escrita la fundamentación de los EVA. Antes se definieron tres categorías a priori a partir del marco teórico (habilidades y aptitudes, roles e interactividad). A continuación se describen las técnicas de recolección de la información utilizadas.

\subsubsection{Revisión documental de los programas de curso}

En esta fase se realizó una revisión documental a partir de los tres pasos propuestos por Bretschneider et al. (2017). Esta revisión se centró específicamente en la fundamentación conceptual de los programas de curso y, por lo tanto, no se consideró en el análisis ni el diseño ni los contenidos alojados en los EVA. Para realizarla, primero se identificaron en los programas de pregrado los cursos relacionados con la integración de las TIC en educación, y luego se solicitaron los planes de estudio de los coordinadores de programa de dichos pregrados. Posteriormente se organizaron los programas de curso por medio de una matriz en una hoja de cálculo, con los siguientes descriptores: Fecha de la versión, Nombre del curso, Licenciatura, Prerrequisito, Correquisito y Ejes temáticos o unidades. Luego se realizó un preanálisis de acuerdo a las tres categorías a priori. En la tabla 1 se presenta un ejemplo de cómo se codificó uno de los cursos en la hoja de cálculo mencionada. 


\section{Tabla 1. Esquema de codificación}

\begin{tabular}{ll}
\hline Fecha & $1 / 02 / 2019$ \\
\hline Nombre del curso & Tecnología de la Información y la Comunicación II \\
\hline Ejes temáticos o unidades & $\begin{array}{l}\text { Se refiere a procesos de enseñanza y aprendizaje, frecuencia de ingreso a la } \\
\text { plataforma (Edmodo), comunicación entre estudiante y profesor }\end{array}$ \\
\hline EVA en la metodología & $\begin{array}{l}\text { Se refiere a ambientes de aprendizaje y actividades complementarias para el } \\
\text { ambiente de aprendizaje }\end{array}$ \\
\hline Roles & Los profesores en formación adquieren el rol de productores de conocimiento \\
\hline Competencias* & $\begin{array}{l}\text { Consonancia con documentos rectores, relación con las competencias digitales } \\
\text { en clave de comunicación y creación de contenido con relación a un EVA }\end{array}$ \\
\hline Interactividad & Digitalización de todo el contenido del curso \\
\hline Inferencias iniciales & $\begin{array}{l}\text { Se observa en el aspecto evaluativo que todos los ítems tiene correlación con } \\
\text { la construcción del "ambiente virtual de aprendizaje”, en el que implícitamente } \\
\text { se puede leer una intención }\end{array}$ \\
\hline
\end{tabular}

Nota: Autoría propia. En la etapa de revisión documental se adoptó el concepto competencias, y luego de las discusiones generadas en las entrevistas se decidió ajustarlo por habilidades y aptitudes.

\subsubsection{Entrevistas semiestructuradas}

Se realizaron entrevistas semiestructuradas para identificar las percepciones y usos de los EVA como espacios complementarios de los profesores. Para su diseño fueron tenidos en cuenta los aportes de Trindade (2016), en particular, en la construcción de las preguntas se tuvo en cuenta que fueran claras, flexibles y de nivel progresivo para los entrevistados. Se identificaron 16 profesores que han orientado los cursos entre 2018 y 2020, y 9 aceptaron participar en la entrevista. Los registros se realizaron por medio de videoconferencia de forma individual siguiendo las indicaciones del código de ética de la institución. En la siguiente lista se muestran las preguntas realizadas a los profesores entrevistados.

1. Desde su experiencia, ¿¿de qué manera entiende un EVA? ¿Incluye EVA en sus cursos y cómo lo hace o haría de ser el caso?

2. ¿Cuál considera que es el potencial educativo para la formación inicial de profesores de los EVA y cómo fomenta o fomentaría su conceptualización a nivel teórico?

3. En su experiencia, ¿cómo considera que son los roles de los estudiantes y profesores al participar en un EVA? ¿se mantienen o varían?
4. Es habitual que algunos de los criterios que se usan para elegir un EVA u otro dependen del potencial identificado en sus funciones y su posibilidad de uso. Por ejemplo, funcionalidad y usabilidad. Desde su experiencia, ¿qué otros criterios o elementos considera relevantes a la hora de elegir e incluir en los entornos virtuales de aprendizaje como espacio complementario en los cursos que orienta?

5. Normalmente, ¿qué aspectos considera o consideraría en la evaluación de los estudiantes cuando participan en los EVA?

6. Desde su experiencia, ¿qué habilidades prioriza o priorizaría en la formación inicial de profesores al desarrollar una propuesta de enseñanza que integre los EVA al currículo?

7. ¿Cómo los EVA pueden fortalecer los procesos de democratización del conocimiento en la formación inicial de profesores?

8. ¿Por qué es prerrequisito o por qué es correquisito? ¿Cree usted que las competencias que adquieren los profesores en formación en este curso, a través de los EVA, aportan al desarrollo de otros espacios de formación? 
9. ¿Qué recomendaciones daría a sus colegas para la integración de EVA en el contexto de la formación de profesores?

10. En este estudio estamos indagando los usos de los EVA como espacios complementarios. ¿Cuáles son algunas consideraciones para implementar estos entornos de aprendizaje como espacio complementario, considerando el contexto actual de la Facultad de Educación?

\subsection{Codificación y categorización}

Luego de la recolección de información se desarrolló el proceso de codificación por medio de una matriz por categoría (habilidades y aptitudes, roles, interactividad y la categoría emergente potencial de los EVA) en la que los cuatro investigadores realizaron la lectura de las 9 entrevistas, identificando así unidades de registro (UR) relacionadas a las categorías a priori. Luego, se organizaron las UR conforme se presenta en la tabla 2 .
Las transcripciones de las entrevistas y los programas de curso fueron analizadas bajo el enfoque exploratorio y descriptivo del análisis de contenido. El análisis de contenido es una forma de considerar la información y los datos textuales de manera sistemática y objetiva, en la cual se presta atención principalmente a las ideas expresadas en el contenido.

Después de establecer las categorías y códigos, se analizó la información recolectada de los programas de los cursos y las entrevistas, para profundizar sobre los EVA y sus características como espacios complementarios.

Luego se procedió a la fase descriptiva y analítica. Al tener el sistema de codificación organizado y un sistema categorial definido, se hizo la selección final y se procedió al análisis. Por último, se realizó la fase interpretativa. Aquí se analizó y comparó el contenido según las categorías a priori y las categorías emergentes referidas a las proyecciones de usos de los EVA como espacios complementarios en los cursos relacionados con la integración de las TIC en educación.

Tabla 2. Codificación de las entrevistas semiestructuradas

\begin{tabular}{|c|c|c|c|}
\hline \multirow{8}{*}{$\begin{array}{l}\text { Categorías a } \\
\text { priori }\end{array}$} & & Código & Descriptor de unidades de registro (UR) \\
\hline & \multirow[b]{2}{*}{$\begin{array}{l}\text { Habilidades } \\
\text { y aptitudes }\end{array}$} & Habilidades técnicas & $\begin{array}{l}\text { Relaciona las UR que aluden a habilidades técnicas y tecnológicas } \\
\text { necesarias para los procesos educativos en los EVA. }\end{array}$ \\
\hline & & $\begin{array}{l}\text { Habilidades educa- } \\
\text { tivas }\end{array}$ & $\begin{array}{l}\text { Corresponde a las UR que especifican aptitudes de los profesores } \\
\text { que orientan los cursos y que posibilitan los EVA en FIP con rel- } \\
\text { ación a los procesos de enseñanza y aprendizaje. }\end{array}$ \\
\hline & \multirow{3}{*}{ Roles } & Rol Profesor & $\begin{array}{l}\text { UR relacionadas con las posibilidades de interacción de profesores } \\
\text { que orientan los cursos. }\end{array}$ \\
\hline & & $\begin{array}{l}\text { Rol profesor en for- } \\
\text { mación }\end{array}$ & UR que referencian el rol del profesor en formación en los EVA. \\
\hline & & Rol EVA & $\begin{array}{l}\text { UR que referencian el papel del EVA y las posibilidades de interac- } \\
\text { ción entre los profesores y el profesor en formación. }\end{array}$ \\
\hline & \multirow{2}{*}{$\begin{array}{l}\text { Interactivi- } \\
\text { dad }\end{array}$} & $\begin{array}{l}\text { Navegabilidad / } \\
\text { funcionalidad }\end{array}$ & $\begin{array}{l}\text { Mención de los criterios técnicos de la plataforma que posibilitan la } \\
\text { usabilidad del EVA. }\end{array}$ \\
\hline & & $\begin{array}{l}\text { Interactividad inmer- } \\
\text { siva/no inmersiva }\end{array}$ & $\begin{array}{l}\text { Referencias en las UR a los niveles de interactividad que posibil- } \\
\text { itan la manipulación del contenido y la construcción de rutas de } \\
\text { aprendizaje autónomas. }\end{array}$ \\
\hline $\begin{array}{l}\text { Categoría } \\
\text { emergente }\end{array}$ & \multicolumn{2}{|c|}{ Potencial de los EVA } & $\begin{array}{l}\text { Posibles rutas de los procesos educativos que usan EVA como } \\
\text { espacios complementarios. }\end{array}$ \\
\hline
\end{tabular}

Nota: Autoría propia. 


\section{Resultados}

Además de las categorías declaradas, el proceso de análisis tuvo como consecuencia la emergencia de una nueva categoría: potencialidades de los EVA. En adelante se presentan los resultados de cada categoría.

\subsection{Habilidades y aptitudes}

Esta categoría se refiere a las habilidades que se privilegian con relación a la proyección de uso de EVA en la FIP. El análisis de los programas de curso permite el énfasis que ponen los estudiantes y profesores en la búsqueda de información, recursos y los derechos de autor. Por su parte, las características de los dispositivos representan uno de los focos de atención de los entrevistados, así como sus beneficios y limitaciones. Otro aspecto son las habilidades técnicas para identificar las herramientas web que permiten hacer una búsqueda adecuada de la información (bases de datos, repositorios, diversos buscadores). Al respecto, el Profesor 3 señala:

[...] por supuesto, también necesitarán alguien que les enseñe cómo funciona esa plataforma y cómo usarla adecuadamente, yo creo que serían esos los elementos [...] sería más que todo sobre eso en relación a las competencias informacionales para profes y estudiantes (Profesor 3, 9/9/2020, entrevista).

Otro aspecto recurrente en el análisis de los programas de curso indica la relevancia de fomentar en la FIP habilidades para identificar las características, fortalezas y debilidades de cada una de las opciones y alternativas que brindan los EVA. Se destacan los componentes comunicacionales y la creación de contenidos para construir conocimiento a través de los EVA, tales como: la evaluación, las habilidades de trabajo, el aprendizaje colaborativo que permite la distribución del trabajo, y la asignación de tareas y compromisos. Al respecto, las competencias de gestión se presentan como fundamentales. Estos resultados coinciden con los registros obtenidos en las entrevistas; por ejemplo, el Profesor 5 manifestaba:

[...] no tiene sentido que el profesor intente implementar unas herramientas, pero no sepa cómo se manejan y una cuestión administrativa o de gestión de cómo dominar o cómo debo trabajar en estos espacios de aprendizaje que insisto no son lo mismo que la presencialidad (Profesor 5, 14/9/2020, entrevista).

En los cursos de FIP que usan EVA como ECF se identificó que se potencia la capacidad de seleccionar, crear y compartir críticamente los contenidos dispuestos en los EVA. Por otro lado, la apropiación del conocimiento disciplinar y las habilidades de pensamiento crítico sobre el uso de los recursos en estrategias mediadas por los EVA también fueron identificadas como relevantes, además, diversos rasgos en coherencia con los conocimientos educativos que tienen como finalidad cumplir con los objetivos de aprendizaje de temas específicos y la evaluación del proceso educativo.

¿Qué hace que ese recurso sea didáctico? ¿qué hace que ese recurso se pueda integrar en un LMS, para formación, o en un sistema de formación, o en un ambiente virtual o en una página web que he diseñado para hacer mis clases?; entonces la prioridad en ese objetivo es esa, que ellos entiendan qué características y qué requisitos ha de cumplir [...] en función de ser didácticos y de proveer algunos elementos que se constituyan en enseñanza (Profesor $7,7 / 9 / 2020$, entrevista).

Finalmente, con relación a la evaluación del proceso de aprendizaje, se hace referencia a las habilidades adquiridas en la FIP que son valoradas por los profesores de los cursos. Se reconoce la relevancia de la evaluación del proceso y la cualificación de los productos por medio de la integración efectiva del conocimiento disciplinar, educativo y tecnológico que se desarrolla en el curso; además de valorar el aprendizaje autorregulado y autogestionado, como los resultados que demuestren un trabajo colaborativo efectivo.

\subsection{Roles}

La categoría roles se comprende como las posibilidades de interacción entre profesores y estudiantes (en este estudio, los estudiantes son futuros profesores) que se dan en los EVA y como las posibilidades 
de interacción entre profesores y estudiantes y los recursos tecnológicos utilizados. Tanto en los programas de curso como en las entrevistas a los profesores se identificó la necesidad de un rol que medie entre el EVA y los estudiantes por parte del profesor que orienta los cursos. Al respecto, se pone énfasis en nuevas labores en la profesión docente, como gestionar EVA que aporten contenido educativo relevante para los estudiantes y también acompañar sus procesos educativos de manera directa con la finalidad de lograr los objetivos propuestos en los cursos.

La complementariedad que se genera entre el EVA y el trabajo presencial en los cursos marca la necesidad de que los profesores se apropien de un sentido crítico frente a los modos de uso de la tecnología con finalidades educativas. Además, aunque no es explícito en los programas de curso, las entrevistas con los profesores permiten reconocer que en entornos virtuales las relaciones de poder se alteran, debido a que no se puede ejercer un control directo sobre las acciones de los estudiantes, lo que implica que deben desarrollar nuevas maneras de interacción con ellos. Con respecto a lo anterior, se reportó en las entrevistas realizadas que, dadas las características de construcción de conocimiento que se encuentran en los EVA, los profesores tienden a interactuar como pares con sus alumnos.

Por otra parte, se identifica que el rol de los profesores en formación también se modifica al interactuar en un EVA, puesto que se convierten en colaboradores de un espacio académico mediado por la virtualidad. En este sentido, se propicia que los futuros profesores adquieran un rol activo y con autonomía en su proceso de aprendizaje, más aún cuando los programas de curso tienden a proponer compromisos académicos que se elaboran a lo largo del semestre, lo que convierte a los estudiantes en creadores de contenido educativo. Así mismo, se identificó que los profesores en formación tienen la posibilidad de mejorar sus habilidades de comunicación en la web al participar en EVA y en las comunidades de aprendizaje y discusión que se gestan en estos entornos. Sobre lo anterior, el Profesor 1 expresa lo siguiente:
La autonomía es uno de los temas fundamentales cuando se trabaja con tecnología, sobre todo con entornos virtuales de aprendizaje [...] ese rol del estudiante, la capacidad de trabajar autónomamente, de manejar sus tiempos, de comprometerse con una formación en la que no está el docente como inspector del proceso (Profesor 1, 11/09/2020, entrevista).

Con respecto al rol que cumple un EVA como espacio complementario en la FIP, se identificó una tendencia a favorecer los diferentes estilos de aprendizaje, dado que quienes participan en estos entornos tienen la posibilidad de interactuar con contenidos educativos de diversas naturalezas y comunicarse con sus compañeros y profesores en distintos formatos y generar hipervínculos entre ellos, lo cual a su vez permite que sus saberes disciplinares se vean enriquecidos por los recursos que ofrecen estas tecnologías, por ejemplo, textos, audio, videos, imágenes, o apps para móviles, los cuales se registran como estrategias reportadas en algunas de las metodologías en los programas de curso. Por último, se hace necesario mencionar que tanto en los programas de curso como en las entrevistas hay un interés marcado por la formación de profesores que se desempeñen con éxito en el uso de TIC en sus respectivos campos específicos, frente a los desafíos que implica la integración de tecnologías digitales en las aulas de clase en todos los niveles educativos.

\subsection{Interactividad}

Se encontró que los profesores establecen como criterio relevante para seleccionar y construir EVA elementos que permiten la comunicación constante con los profesores en formación, implementando estrategias basadas en la comunicación asincrónica. Los profesores manifiestan la importancia de seleccionar EVA que centren la interactividad en el componente comunicacional desde una conexión bidireccional. También se encuentra como un asunto recurrente contemplar que el EVA posibilite la conexión y correcta visualización del contenido desde diferentes navegadores, equipos de escritorio y dispositivos móviles. En particular, se identifica en los programas de curso la incorporación mayoritaria de plataformas 
como Classroom, de Google, y Moodle, desde los servidores de la universidad. Al respecto, el Profesor 8 menciona las características que deben ofrecer estos entornos:

[...] que tenga unos espacios de comunicación dispuestos para interactuar tanto con el profesor como con sus compañeros, eso es vital; el asunto de la comunicación, pero adicional a eso que tenga una estructura simple, una estructura sencilla de navegación y que tenga dispuesto los materiales organizadamente, que si es por semanas, que si es por módulos, que él tenga muy claro cómo navegar este curso (Profesor 8, 14/9/2020, entrevista).

En cuanto al contenido, se busca que los EVA permitan el diseño con características hipertextuales e hipermediales, que sean editables, configurables y flexibles a la hora de adaptarlos a los intereses y objetivos formativos. Se enfatiza la necesidad de contemplar que tanto el contenido como la plataforma cumplan el principio de accesibilidad, al señalar que la navegación y el menú de ayuda deben cumplir con asistencia técnica que favorezca el acceso inclusivo al EVA.

Respecto a la interactividad contextual no inmersiva, no se reportó su adjetivación en lo señalado por los profesores en las entrevistas; a su vez, tampoco se mencionó en los programas de curso. Sin embargo, sí se señalan algunas características que componen este nivel de interactividad. Entre las más recurrentes se señala la relevancia de elegir EVA que integren herramientas y recursos necesarios en los procesos de autogestión del aprendizaje, y que posibiliten una navegación accesible sobre el contenido que se dispone en la plataforma. En particular, estas características fueron identificadas de forma explícita en las competencias didácticas que se busca que adquieran los profesores en formación reportadas en los programas de curso.

Con relación a la Interactividad Contextual Inmersiva se hallan relaciones incipientes en cuanto a la navegación, a su vez, aparecen unas pocas menciones a la autogestión del contenido. Este tipo de interactividad sugiere que el contenido dispuesta en los EVA sea manipulable, lo que supone un reto en su diseño y en hacer que los estudiantes sean conscientes de dicha manipulación. Sobre este tipo de interactividad, los profesores hacen referencia a herramientas tecnológicas emergentes como la gamificación, la realidad aumentada y la big data.

[...] me parece fundamental hoy pensar en las adecuaciones necesarias para atender a las diferentes capacidades. Los temas de inclusión y calidad deben evaluar cómo esto se puede dar por medio de los entornos virtuales de aprendizaje, posibilitando y evaluando a la hora de seleccionar un entorno que permita la lectura de pantalla, que permita alto contraste para atender a esas diversas necesidades (Profesor 1, 11/9/2020, entrevista).

Sobre los criterios que establecen los profesores para valorar la participación de los estudiantes mediante los EVA, se señala de forma regular la participación en actividades conjuntas que se disponen en la plataforma. Dos de las estrategias señaladas son la participación en los foros y la retroalimentación que se realiza a través de la producción del contenido educativo.

\subsection{Potencialidades de los EVA}

La emergencia de esta categoría atiende a la alta frecuencia en la que surgió la preocupación por las posibles rutas a futuro de los procesos educativos que usan EVA como espacios complementarios. Los profesores entrevistados reconocen en los EVA un amplio potencial educativo frente a los desafíos a los que se enfrenta la educación en la actualidad, más aún en el contexto de la pandemia provocada por el COVID-19. En este sentido, el profesor 6 reporta lo siguiente:

En los procesos que hacemos en el marco de los proyectos nos hemos encontrado que un escenario posible y potencial para favorecer procesos de enseńanza y aprendizaje son los que se basan en las interacciones sociales. Es decir, encontrar un escenario en el que sea fácil para el estudiante, por ejemplo, crear comunidades de aprendizaje, crear comunidades virtuales de aprendizaje donde sea fácil para ellos llegar a acuerdos con sus compañeros y poder comunicarse (Profesor 6, 11/09/2020, entrevista). 
Sin embargo, declaran que el uso de EVA en sí mismo no soluciona los problemas en la educación, más bien consideran que estos entornos cobran relevancia en tanto son gestionados por profesores con recursos tanto metodológicos como teóricos para llevar a cabo procesos educativos exitosos. Al respecto, se tiene en cuenta como aspecto fundamental para alcanzar el potencial educativo de los EVA, desarrollar una cultura de construcción del conocimiento en su interior, en la que se posibilite que todos los participantes compartan contenidos y experiencias a través del trabajo colaborativo de manera tanto sincrónica como asincrónica.

No es el ambiente por sí solo, es lo que hace el profesor con ese ambiente, o es la orientación, o es el interés que tiene el estudiante (Profesor 8, 14/09/2020, entrevista).

Además, los sujetos entrevistados insisten en la posibilidad que presentan los EVA para favorecer distintos estilos y ritmos de aprendizaje, por lo que los profesores en formación encuentran la posibilidad de avanzar en los cursos siguiendo diversas rutas y comparando sus construcciones con las de sus compañeros. De igual modo, se reportaron como significativos frente al potencial educativo de los EVA los elementos que se presentan frente a la democratización del conocimiento. Lo anterior se presenta dado que en la web existen distintas alternativas para la construcción de EVA abiertos para toda la comunidad académica.

\section{Discusión y conclusiones}

\subsection{Discusiones}

Los resultados de este trabajo permitieron encontrar una proyección de uso central de los EVA como ECF frente a la posibilidad de la construcción de conocimiento en la FIP (Manrique-Losada et al., 2020). Por otro lado, la gestión de los contenidos del profesor y la autonomía de los profesores en formación se adhieren a las habilidades metacognitivas propuestas por Cassidy (2016). En este sentido, las habilidades metacognitivas resultan transversales en tanto reflejan la posibilidad de potenciar las aptitudes de autogestión y regulación del aprendizaje (Zangara, 2018). Frente a lo anterior, la investigación resalta la percepción de los docentes que señala la autonomía como una habilidad a priori que poseen los profesores en formación. Sin embargo, estudios como los de Manrique-Losada et al. (2020) y Sutcliffe y Hart (2017) la resaltan como una habilidad que se fortalece en los procesos educativos que son mediados por las TIC.

Por otro lado, el desarrollo de nuevas habilidades como la búsqueda de información y la integración de recursos en los EVA con finalidad educativa se relaciona con las habilidades de previsión del proceso de enseñanza y aprendizaje propuestas por García-Cabrero et al. (2018). Sin embargo, se encuentran tensiones referentes al propósito del EVA con relación a los objetivos formativos de los cursos, lo cual puede desembocar en una disociación entre el potencial del EVA y su uso como ECF (Arroyo-Vera et al., 2018). Además, es necesario que el profesor medie sobre la apropiación tecnológica de los participantes del entorno (Phungsuk et al., 2017).

Por otro lado, las relaciones entre los distintos participantes se desarrollan de manera horizontal, según estudios como el de Granados et al. (2017) que señala la posibilidad de fomentar el trabajo colaborativo a través de relaciones horizontales. Además, otras perspectivas como las de Cassidy (2016) sostienen que la figura del profesor continúa siendo fundamental para gestionar tanto los contenidos básicos del curso que se insertan en la plataforma, como los que los profesores en formación construyen y comparten en el proceso formativo.

En este sentido, se identifica un desafío respecto al reconocimiento y elección de las diversas opciones metodológicas que surgen en los EVA y los roles en el proceso. Por otra parte, son relevantes las relaciones que se posibilitan entre los profesores que orientan los cursos y los profesores en formación con relación a la comunicación asincrónica (Echeverry \& Jaramillo, 2017). En contraste con estudios realizados por Arias et al. (2014) que proponen el rol del profesor como mediador entre la plataforma y el estudiante en 
términos de la transmisión del conocimiento y las posibilidades reales que tienen los estudiantes de diseñar su propio proceso de aprendizaje.

Por último, con respecto a los aspectos técnicos involucrados en los EVA, los criterios de usabilidad más recurrentes son la capacidad de la plataforma para potenciar la comunicación, la facilidad de navegación y la operatividad, como lo proponen Díaz-Gutiérrez y Valderrama-García (2018); además, se identifica una preocupación sobre las posibles dificultades de conectividad, técnicas y de accesibilidad que impidan el correcto desarrollo de actividades de los EVA. Dicha preocupación se adhiere a lo propuesto por Badilla et al. (2017) y converge con lo hallado por Veraszto y García (2011). Aparte de ello, Fainholc (2016) señala la relevancia de ubicar dichas características tanto en el EVA como en los contenidos y recursos integrados, estos elementos derivan niveles de interactividad contextual no inmersiva al posibilitar una manipulación y trasformación del contenido del EVA.

\subsection{Conclusiones}

Este estudio buscó describir las proyecciones de usos de EVA como ECF en cursos de TIC de los programas de FIP de la Facultad de Educación de la Universidad de Antioquia, por medio de una revisión documental de los programas de curso y entrevistas semiestructuradas a los profesores responsables de los cursos, a partir de cuatro categorías (roles, habilidades y actitudes, interactividad y potencial de los EVA).

Se encuentran proyecciones de usos heterogéneos con relación a la naturaleza del curso, la concepción y percepción de los EVA sobre el potencial de las TIC en los procesos educativos. Como hallazgo, se encontró una proyección de uso continuo explícito o intuitivo de los EVA, donde se articula la virtualidad como ECF desde las diferentes prácticas disciplinarias y educativas.

Si bien se reportó que el desarrollo de procesos educativos mediados por los EVA fomenta la autogestión del aprendizaje, también se evidencia que, para un desarrollo de procesos educativos exitosos en un EVA, es necesario que estas habilidades estén ya incorporadas a priori en los futuros profesores. De la misma manera, dado que los EVA posibilitan la consolidación de rutas de aprendizaje personalizadas por los estudiantes, surge un reto en cuanto al diseńo de experiencias de aprendizaje que consideren niveles de interactividad contextual inmersiva para favorecer la manipulación y transformación del contenido en tiempo real, dicha manipulación del contenido posibilita la construcción de conocimiento.

Por otra parte, las potencialidades reportadas por la comunidad educativa son amplias, los EVA pueden integrar tecnologías emergentes en los contextos educativos para desarrollar procesos metodológicos y tecnológicos. Para ello es necesario fomentar la consolidación de comunidades de conocimientos que dinamicen los EVA como espacio complementario a la presencialidad.

Por último, los resultados de este estudio permiten proyectar futuras investigaciones, en las cuales será necesario considerar las características necesarias para desarrollar EVA con los recursos educativos pertinentes para la especificidad de los diversos saberes que confluyen en la Facultad de Educación (p. ej. matemáticas, literatura, educación especial).

\section{Agradecimientos}

Al Centro de Investigaciones Educativas y Pedagógicas (CIEP) de la Facultad de Educación de la Universidad de Antioquia (Colombia), por la financiación del proyecto de investigación "Implementación de los entornos virtuales de aprendizaje como espacio complementario en programas de pregrado de la Facultad de Educación de la Universidad de Antioquia”.

\section{Referencias bibliográficas}

Alvarez, I., Guasch, T., \& Espasa, A. (2009). University teacher roles and competencies in online learning environments: a theoretical analysis of teaching and learning practices. European Journal of Teacher 
Education, 32(3), 321-336.

https://doi.org/10.1080/02619760802624104

Arbeláez, M., \& Onrubia, J. (2014). Análisis bibliométrico y de contenido. Dos metodologías complementarias para el análisis de la revista colombiana Educación y Cultura. Revista de Investigaciones UCM, 14(23), 14-31.

http://dx.doi.org/10.22383/ri.v14i1.5

Arias, M., Torres, T., \& Yáñez, J. C. (2014). El desarrollo de competencias digitales en la educación superior. Historia y Comunicación Social, 19, 355-366. https://doi.org/10.5209/rev_HICS.2014.v19.44963

Arroyo-Vera, Z., Fernández-Prieto, M. S., BarretoZambrano, L., \& Paz-Enrique, L. E. (2018). Entornos virtuales de aprendizaje en comunidades de práctica de docentes universitarios del Ecuador. Ensayos Pedagógicos, 13(2), 185-200.

https://r.issu.edu.do/l?l=10254Dwb

Ayil-Carrillo, J. S. (2018). Entorno virtual de aprendizaje: una herramienta de apoyo para la enseñanza de las matemáticas. Revista de Investigación en Tecnologias de la Información: RITI, 6(11), 34-39.

https://r.issu.edu.do/l?1=10242sop

Badilla, M. G., Sagredo, A., \& Lytras, M. D. (2017). Pre-service teachers' skills and perceptions about the use of virtual learning environments to improve teaching and learning. Behaviour \& Information Technology, 36(6), 575-588.

https://doi.org/10.1080/0144929X.2016.1266388

Borba, M. C., de Souza Chiari, A., \& de Almeida, H. R. F. L. (2018). Interactions in virtual learning environments: new roles for digital technology. Educational Studies in Mathematics, 98(3), 269-286. https://doi.org/10.1007/s10649-018-9812-9

Bretschneider, P. J., Cirilli, S., Jones, T., Lynch, S., \& Wilson, N. A. (2017). Document review as a qualitative research data collection method for teacher research. SAGE Publications Ltd.

https://dx.doi.org/10.4135/9781473957435

Carmona, E. J., \& Rodríguez, E. (2017). Buenas prácticas en la educación superior virtual a partir de especificaciones de estándares e-Learning. Sophia, 13(1), 13-26.

http://dx.doi.org/10.18634/sophiaj.13v.1i.345

Cassidy, S. (2016). Virtual learning environments as mediating factors in student satisfaction with teaching and learning in higher education. Journal of Curriculum and Teaching, 5(1), 113-123.

https://doi.org/10.5430/jct.v5n1p113

Durak, H. Y. (2019). Examining the acceptance and use of online social networks by preservice teachers within the context of unified theory of acceptance and use of technology model. Journal of Computing in Higher Education, 31(1), 173-209.

https://doi.org/10.1007/s12528-018-9200-6

Díaz-Gutiérrez, E. L. \& Valderrama-García, C. F. (2018). Evaluación de la usabilidad de los EVA (entornos virtuales de aprendizaje) a partir de la experiencia de usuarios aplicando lógica difusa. Revista Vinculos: Ciencia, tecnología y sociedad, 15(2), 150159. https://doi.org/10.14483/2322939X.14006

Echeverry, J., \& Jaramillo, M. (2017). La relación profesor-estudiante en los programas de educación virtual [Tesis de maestría Universidad de Antioquia, Medellín].

http://bibliotecadigital.udea.edu.co/bitstream/10495/9863/1/ EcheverryJuan_2017_RelacionProfesorEstudiante.pdf

Fainholc, B. (2016). Presente y futuro latinoamericano de la enseńanza y el aprendizaje en entornos virtuales referidos a educación universitaria. Revista de Educación a Distancia (RED), 48.

https://r.issu.edu.do/l?l=10234NBU

Flick, U. (2014). La gestión de la calidad en investigación cualitativa. Madrid: Morata.

García-Cabrero, B., Luna-Serrano, E., Ponce-Ceballos, S., Cisneros-Cohenour, E., Cordero-Arroyo, G., Espinoza-Díaz, Y., \& García-Vigil, M. H. (2018). Las competencias docentes en entornos virtuales: Un modelo para su evaluación. Revista Iberoamericana de Educación a Distancia, 21(1), 343-365.

https://r.issu.edu.do/l?l=10302gMd 
González, K., Padilla, J., \& Rincón, A. (2013). Las competencias docentes en entornos virtuales: Un modelo para su evaluación. Revista Virtual, (36), 4874. https://r.issu.edu.do/l? $1=102402 \mathrm{zl}$

Granados, J. F., Vargas-Pérez, C., \& López-Fernández, R. (2017). Estrategia de formación continua del docente universitario en la didáctica de los entornos virtuales de aprendizaje (EVA). Revista Conrado, 13(1-Ext), 78-86.

https://r.issu.edu.do/l?l=1023845Z

Hrastinski, S. (2019). What Do We Mean by Blended Learning? TechTrends, 63(5), 564-569.

https://doi.org/10.1007/s11528-019-00375-5

Hue Dung, D. T. (2020). The advantages and disadvantages of virtual learning. IOSR Journal of Research \& Method in Education, 10(3), 45-48.

https://r.issu.edu.do/l? l=10237g2x

Lai, E., \& Xue, Y. (2012). On the Influence of online education on teacher-student relationship. In Soft Computing in Information Communication Technology (pp. 49-55). Berlin: Springer.

Llorente-Cejudo, M., \& Cabero A. J. (2008). Del eLearning al Blended Learning: nuevas acciones educativas. Quaderns Digitals: Revista de Nuevas Tecnologias y Sociedad, 51, 30.

https://r.issu.edu.do/l?l=10243sUx

Manrique-Losada, B., Zapata-Cárdenas, M. I., \& Arango-Vásquez, S. I. (2020). Entorno virtual para cocrear recursos educativos digitales en la educación superior. Campus Virtuales, 9(1), 101-112.

https://r.issu.edu.do/l? l=10246Ch

Rodríguez-Ortiz, A. M., \& Sosa-Neira, E. A. (2018). Interactividad e interacción social: procesos esenciales en educación a distancia. Revista Virtual Universidad Católica del Norte, 55, 110-127.

https://r.issu.edu.do/l?1=10301KSH
Phungsuk, R., Viriyavejakul, C., \& Ratanaolarn, T. (2017). Development of a problem-based learning model via a virtual learning environment. Kasetsart Journal of Social Sciences, 38(3), 297-306. https://doi.org/10.1080/14675986.2017.1333874

Quiroz-Vallejo, D. A., Clavijo-Gallo, C., Díaz-Ruiz, M., \& Carmona-Mesa, J. A. (2020). ¿Facebook para la formación de profesores? Una revisión de la literatura. Revista Espacios, 41(28), 71-84.

https://r.issu.edu.do/l?l=10232ev4

Sutcliffe, A., \& Hart, J. (2017). Analyzing the role of interactivity in user experience. International Journal of Human-Computer Interaction, 33(3), 229-240. https://doi.org/10.1080/10447318.2016.1239797

Trindade, V. A. (2016). Entrevistando en investigación cualitativa y los imprevistos en el trabajo de campo: de la entrevista semiestructurada a la entrevista no estructurada. En Schetini, Técnicas y estrategias en la investigación cualitativa (pp. 18-32). Editorial Universidad de la Plata.

https://r.issu.edu.do/l?l=10304JbG

Veraszto, E. V., \& García, F. (2011). Interatividade e educação: reflexóes acerca do potencial educativo das TIC. Interciência \& Sociedade, Mogi Guaçu: Faculdade Municipal Franco Montoro, 1(1), 85-96.

https://r.issu.edu.do/l?1=10251rlN

Vixtha-Vázquez, F. (2017). Interactividad y multimedialidad: elementos que la hipermediación aporta a la comunicación educativa. Razón y Palabra, 21(398), 206-220.

https://r.issu.edu.do/l?l=10303hTT

Zangara, M. A. (2018). Interacción e interactividad en el trabajo colaborativo mediado por tecnología informática [Tesis doctoral, Universidad Nacional de La Plata, Argentina]. https://r.issu.edu.do/l?1=102495dV 
Revista Caribeña de Investigación Educativa | 2021, 5(2), 92-106

\section{CÓMO CITAR:}

Clavijo-Gallo, C., Quiróz-Vallejo, D. A., Arboleda-Mira, E. J., \& Carmona-Mesa, J. A. (2021). Entornos virtuales de aprendizaje como espacio complementario en la formación inicial de profesores. RECIE. Revista Caribeña de Investigación Educativa, 5(2), 92-106. https://doi.org/10.32541/recie.2021.v5i2.pp92-106 